\title{
Autologous Breast Reconstruction Skin Paddle Designs: Classification and Aesthetic Outcomes
}

\author{
Ivo A. Pestana, MD ${ }^{1}$ Nicholas J. Walker, MD ${ }^{1}$ \\ ${ }^{1}$ Department of Plastic and Reconstructive Surgery, Wake Forest \\ Baptist Health, Winston Salem, North Carolina \\ J Reconstr Microsurg Open 2019;4:e29-e35.
}

\begin{abstract}
Address for correspondence Ivo A. Pestana MD, Department of Plastic and Reconstructive Surgery, Wake Forest Baptist Health, 1 Medical Center Boulevard, Winston Salem, 27157, NC (e-mail: ipestana@wakehealth.edu).
\end{abstract}

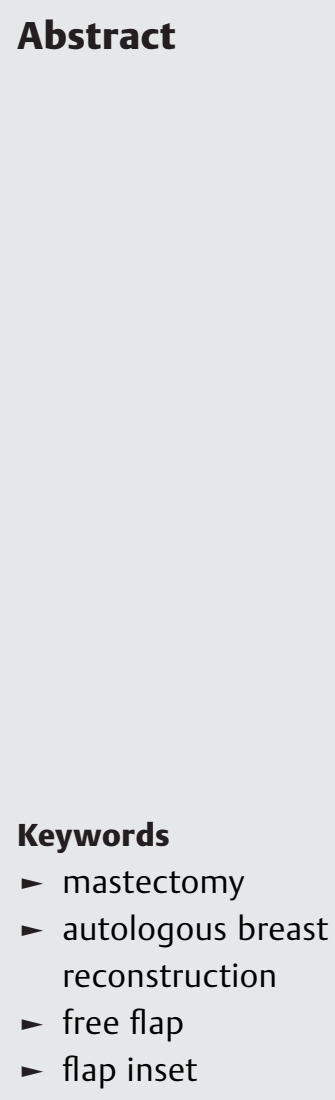

Objective Present study was conducted to identify common mastectomy patterns and autologous skin paddle designs, to identify patient characteristics that influence these patterns, and assess aesthetic outcomes associated with each pattern.

Methods Autologous breast reconstructions over 5 years were reviewed. Mastectomy type and resultant skin paddle were categorized into four mastectomy type/skin paddle design groups based on the amount of native "Skin Spared" after mastectomy and the resultant flap skin paddle designed. Groups were designated as skin-sparing mastectomy 1 (SS1)/large elliptical skin paddle, skin-sparing mastectomy 2 (SS2)/small elliptical skin paddle, skin-sparing mastectomy 3 (SS3)/areola skin paddle, and skinsparing mastectomy 4 (nipple-sparing mastectomy, SS4)/no skin paddle. Surveys were performed to validate the classification system and critique aesthetic outcomes.

Results A total of 89 autologous breast reconstructions were included. Radiotherapy was used in $45.6 \%$ of SS1 patients versus $29.2 \%$ in SS2 and $12.5 \%$ in SS3/SS4. Mean body mass index (BMI) was 30 in SS1/SS2 and 26 in SS3/SS4 mastectomy types ( $p=0.045$ ). Delayed reconstruction was performed in $96.5 \%$ SS1 versus $62.5 \%$ in SS2 and only $25 \%$ of SS3/SS4 ( $p<0.0001)$. Physicians and Non-MD personnel correctly categorized 85.8 and $76.1 \%$ of skin paddle designs, respectively. Over $75 \%$ of those surveyed rated the reconstruction aesthetic outcome as "good-excellent" regardless of the pattern group. Conclusions Patients in SS1/SS2 groups had a higher incidence of radiotherapy, delayed reconstruction, and higher BMI compared with the SS3/SS4 groups. The classification system is recognizable and may provide improved patient education and communication between healthcare providers. All mastectomy type/skin paddle designs received high aesthetic ratings.
Breast reconstruction following mastectomy is associated with a positive influence on a patient's breast appearance, psychosocial status, and sexual well-being. ${ }^{1,2}$ Use of autogenous soft tissues for breast reconstruction is increasing for several reasons. This reconstruction technique has an acceptable risk profile and produces a natural appearing breast. ${ }^{3}$ In addition, autologous tissue allows for improved symmetry with a contralateral native breast in unilateral mastectomy patients. ${ }^{4}$ Most recently, concerns regarding implant reconstruction have surfaced stimulating re-evaluation of surgical interventions for implant complications including the occurrence of implant associated lymphomas. 5,6

Autologous breast reconstruction is distinct from implant reconstruction because it provides both breast volume and may add new skin to the breast in the form of a skin paddle. The skin paddle serves multiple purposes including replacing resected native skin and paddle examination is the most sensitive manner of flap perfusion monitoring. The balance of native skin envelope and newly added skin paddle varies and this ratio results in predictable patterns of the reconstructed received

October 22, 2018 accepted after revision March 18, 2019
DOI https://doi.org/

10.1055/s-0039-1688725. ISSN 2377-0813.
Copyright $\odot 2019$ by Thieme Medical Publishers, Inc., 333 Seventh Avenue, New York, NY 10001, USA. Tel: +1(212) 584-4662.
License terms

(요 (1) $\circledast$ 
breast. Several studies discuss techniques to improve the aesthetic outcomes of autologous breast reconstruction but, to our knowledge, none address the influence of the mastectomy pattern on autologous skin paddle design. 7,8

This study was conducted to identify common mastectomy patterns and define their effect on autologous flap skin paddle design in an attempt to create a standardized nomenclature to assist in communication amongst providers and with patients, to identify patient characteristics that influence which pattern is ultimately selected in autologous breast reconstruction, and to assess aesthetic outcomes associated with each pattern.

\section{Methods}

An institutional review board (IRB)-approved retrospective review of autologous breast reconstructions performed by a single surgeon over 5 years was completed. Patients ages 18 to 99 who underwent autologous breast reconstruction after mastectomy were included. Patients undergoing lumpectomy reconstruction, oncoplastic breast surgery, and latissimus dorsi myocutaneous (LDMC) flap reconstructions were excluded. Factors, including mastectomy technique, reconstruction technique, use of perioperative chemoradiation, timing of reconstruction, age at time of reconstruction, body mass index (BMI), smoking status, and comorbidities, were evaluated. The incidence of complications was examined and included partial or complete flap loss, hematoma/seroma, fat necrosis, and infection.

Standard patient photographs (preoperative and postoperative) were examined for each patient and their mastectomy type and resultant skin paddle design was categorized into one of four groups based on the amount of native skin present from the mastectomy and the resultant flap skin paddle designed. The mastectomy type/skin paddle groups were designated as skin-sparing mastectomy 1 (SS1)/large elliptical skin paddle, skin-sparing mastectomy 2 (SS2)/small elliptical skin paddle, skin-sparing mastectomy 3 (SS3)/areola skin paddle, and skin-sparing mastectomy 4 (nipple-sparing mastectomy, SS4)/no skin paddle (-Fig. 1).

Our first survey was performed to validate the classification system. Thirty medical doctors (MD) and 22 non-MD personnel (NMD) were asked to categorize randomized pictures of patients who had undergone mastectomy and autogenous reconstruction into their respective groups. Each survey participant was given a standardized explanation and cartoon image as well as example patient images of the mastectomy/ skin paddle groups ( $\mathbf{- F i g . ~ 2 ) , ~ s u b s e q u e n t l y ~ a d m i n i s t e r e d ~ t h e ~}$ survey, and their results recorded. These same participant groups were then administered a second survey to critique the aesthetic outcomes of each mastectomy group. The participants were administered a five question, Likert's scale survey that presented randomized pictures of patients following mastectomy and autogenous reconstruction and asked them to grade each on important aesthetic parameters of the reconstructed breast. This included rating the scar appearance on the final reconstructed breast, the overall size of the skin paddle, the influence of the skin paddle position on scar appearance and in relation to the nipple areolar complex (NAC), and the overall appearance of the reconstructed breast. Their results were recorded and analyzed for trends.

Statistical analysis was performed to evaluate trends and differences between groups. Continuous measures were compared with independent $t$-tests or analysis of variance (ANOVA) tests. For categorical measures, Fisher's exact tests were employed. A $p$-value of $\leq 0.05$ was used to indicate statistical significance.

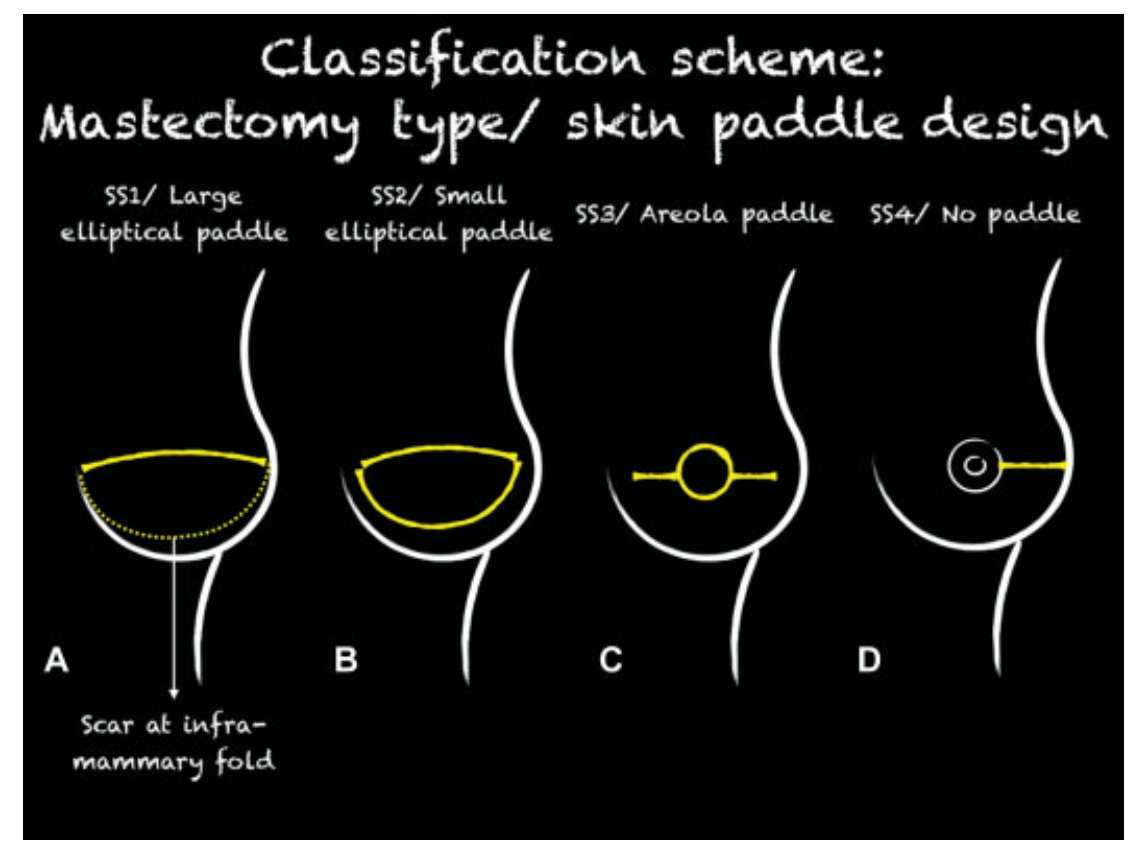

Fig. 1 Mastectomy type and their resultant skin paddle designs. (A) SS1/large elliptical paddle: large skin paddle with a transverse superior scar and an inferior scar hidden in the inframammary fold. (B) SS2/small elliptical paddle: central elliptical skin paddle on the anterior surface of the breast mound, all scars visible on the breast surface. (C) SS3/areola paddle: small central circular skin paddle located at the planned nipple areolar complex position. (D) SS4/no paddle: nipple sparing mastectomy with lateral radial and periareolar scar. 


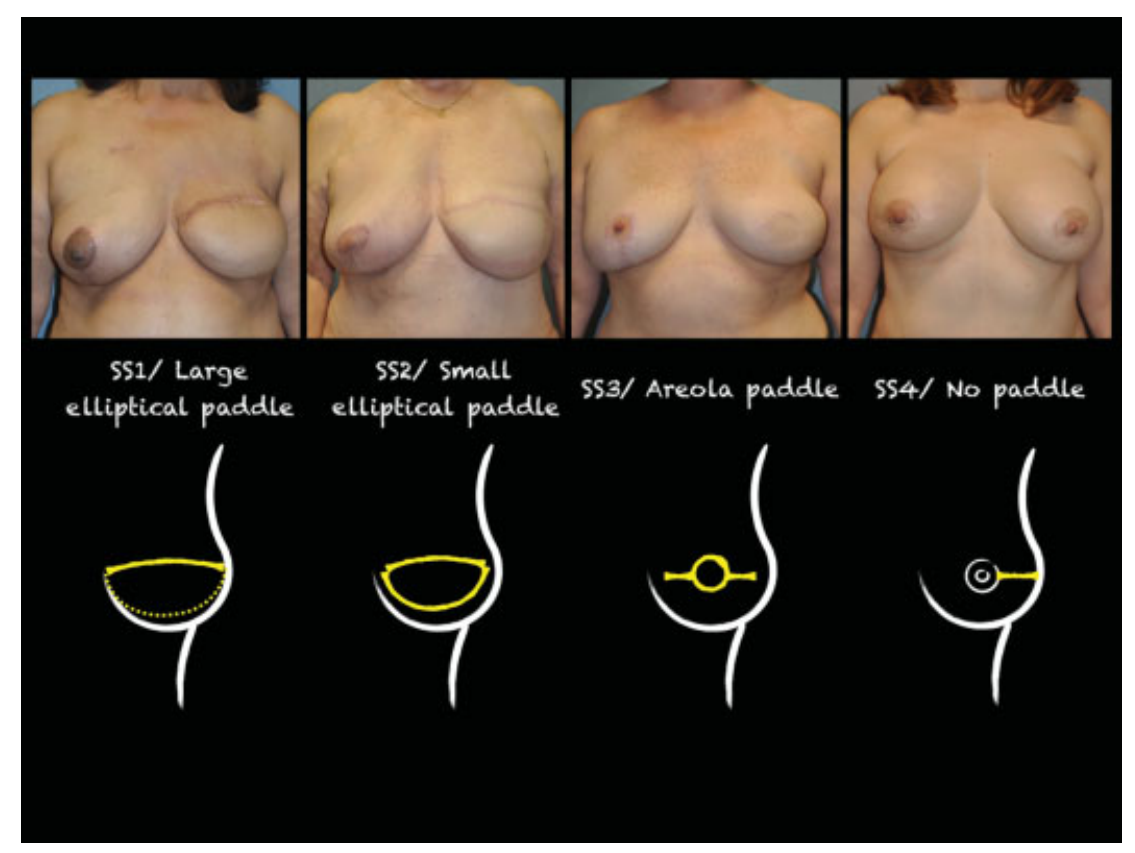

Fig. 2 Mastectomy pattern classification scheme. These classifications are based on final scar and skin paddle position in relationship to the reconstructed breast mound.

\section{Results}

A total of 89 autologous breast reconstructions were performed on 71 female patients. There were 57 breasts categorized as SS1 mastectomy/large elliptical skin paddle, 24 breasts as SS2 mastectomy/small elliptical skin paddle, seven breasts as SS3 mastectomy/areolar skin paddle, and one as SS4 mastectomy/no skin paddle. Flap types employed included four pedicled Transverse rectus abdominus myocutaneous (pTRAM) flaps, 49 muscle-sparing free TRAM (ms fTRAM) flaps, 32 deep inferior epigastric artery perforator (DIEP) flaps, three superficial inferior epigastric artery (SIEA) flaps, and one transverse upper gracilis (TUG) flap. The mean patient age at the time of surgery was $48.8 \pm 9.1$ years with a range of 29 to 73 years. One patient in our study underwent bilateral mastectomy for treatment of chronic mastitis. All other patients underwent mastectomy for treatment of breast cancer or cancer prophylaxis. Cancer diagnoses included 51 patients with invasive ductal carcinoma, eight with invasive lobular carcinoma, nine with ductal carcinoma in situ, one with Paget's disease with intraductal carcinoma, and one patient who underwent prophylactic mastectomy for BRCA gene positivity. Mastectomy types utilized were 17 modified radical, 53 simple/total, and one nipple-sparing mastectomy. Modified radical mastectomy was employed in $37 \%(n=21)$ of breasts in the SS1 mastectomy group and $17 \%$ $(n=4)$ of breasts in the SS2 group. There was no significant difference in incidence of operative complication rates or incidence/type of medical comorbidities between all four mastectomy/skin paddle groups. Prereconstruction radiation was employed in $38.2 \%(n=34)$ of patients. Radiotherapy use in each of the mastectomy groups was $46 \%$ $(n=26)$ of SS1, 29\% $(n=7)$ of SS2, and $13 \%(n=1)$ of SS3/SS4 patients, which did not reach statistical significance $(p=0.111)$. Mean BMI was $30 \pm 4 \mathrm{~kg} / \mathrm{m}^{2}$ in SS1, $30 \pm 5 \mathrm{~kg} /$ $\mathrm{m}^{2}$ in SS2, and $26 \pm 2 \mathrm{~kg} / \mathrm{m}^{2}$ in SS3/SS4 mastectomy patients, respectively, and this difference was statistically significant $(p=0.045)$. Delayed reconstruction was performed in $96.5 \%$ $(n=55)$ of SS1 patients compared with $62.5 \%(n=15)$ in SS2 patients. Immediate breast reconstruction was performed in $75 \%(n=6)$ of SS3/SS4 mastectomy patients, and the difference in timing of reconstruction between groups reached statistical significance ( $p<0.0001 ;$ - Table 1$)$.

A total of $30 \mathrm{MD}$ and 22 non-MD personnel participated in the mastectomy group categorization and aesthetic surveys. Physicians correctly categorized patients into their respective mastectomy groups $85.8 \%$ of the time versus $76.14 \%$ correct answers from non-MD personnel.

In our aesthetic survey, over 75\% of NMDs and MDs rated the overall aesthetic outcomes of the reconstruction as "goodexcellent" regardless of the mastectomy/skin paddle group. With regard to skin paddle size, the large and small elliptical paddle groups had three to five times higher rate of "somewhat to extremely large" rating compared with that of the areolaand no skin paddle groups, respectively, by both MD and nonMD personnel. Over $60 \%$ of MDs felt that the areola and no skin paddle groups were "perfect size" versus $56 \%$ and $46 \%$ by NMD, respectively. Sixty percent of MDs found that the elliptical skin paddles were "somewhat distracting to the nipple areolar complex" or "ideally positioned around the nipple areolar complex, but noticeable" while 55\% of non-MD personnel found them "very good, blends in naturally with NAC/barely noticeable" to "excellent/native appearance around NAC." Overall, both MDs and NMDs found the no skin paddle group to have the highest rates of "excellent/native appearance around NAC." MDs marked this answer almost twice as often for the no skin paddle group compared with the large elliptical paddle and NMDs marked this answer over thrice more often 
Table 1 Patient demographics, complication rates, and procedures performed

\begin{tabular}{|c|c|c|c|c|c|c|c|c|c|}
\hline & \multicolumn{2}{|c|}{ Overall } & \multicolumn{2}{|c|}{ SS1 $(n=57)$} & \multicolumn{2}{|c|}{ SS2 $(n=24)$} & \multicolumn{2}{|c|}{$\begin{array}{l}\text { SS3/SS4 } \\
(n=8)\end{array}$} & $p$-Value \\
\hline Age at date of surgery (y) & \multicolumn{2}{|c|}{$48.83 \pm 9.07$} & \multicolumn{2}{|c|}{$47.54 \pm 9.09$} & \multicolumn{2}{|c|}{$52.17 \pm 9.24$} & \multicolumn{2}{|c|}{$48.00 \pm 6.44$} & 1.07 \\
\hline Body mass index $\left(\mathrm{kg} / \mathrm{m}^{2}\right)$ & \multicolumn{2}{|c|}{$29.92 \pm 4.59$} & \multicolumn{2}{|c|}{$30.27 \pm 4.26$} & \multicolumn{2}{|c|}{$30.35 \pm 5.43$} & \multicolumn{2}{|c|}{$26.09 \pm 2.01$} & $0.045^{\mathrm{a}}$ \\
\hline & $n$ & $\%$ & $n$ & $\%$ & $n$ & $\%$ & $N$ & $\%$ & \\
\hline Perioperative chemo therapy & 27 & 30.34 & 19 & 33.33 & 6 & 25 & 2 & 25 & 0.7141 \\
\hline Perioperative radiation & 34 & 38.2 & 26 & 45.61 & 7 & 29.17 & 1 & 12.5 & 0.1111 \\
\hline Prior history of smoking ${ }^{b}$ & 11 & 12.36 & 10 & 17.54 & 0 & 0 & 1 & 12.5 & 0.0908 \\
\hline \multicolumn{10}{|l|}{ Comorbidities } \\
\hline Diabetes mellitus type 2 & 6 & 6.74 & 2 & 3.51 & 4 & 16.67 & 0 & 0 & 0.0711 \\
\hline Hypertension & 28 & 32.18 & 16 & 28.07 & 10 & 45.45 & 2 & 25 & 0.3003 \\
\hline Peripheral vascular disease & 0 & 0 & 0 & 0 & 0 & 0 & 0 & 0 & - \\
\hline \multicolumn{10}{|l|}{ Timing of reconstruction } \\
\hline Delayed & 72 & 80.9 & 55 & 96.49 & 15 & 62.5 & 2 & 25 & \multirow[t]{2}{*}{$<0.0001^{\mathrm{a}}$} \\
\hline Immediate & 17 & 19.1 & 2 & 3.51 & 9 & 37.5 & 6 & 75 & \\
\hline \multicolumn{10}{|l|}{ Procedure type } \\
\hline DIEP & 32 & 35.96 & 22 & 38.6 & 7 & 29.17 & 3 & 37.5 & \multirow[t]{3}{*}{0.7273} \\
\hline SIEA or TUG & 4 & 4.49 & 2 & 3.51 & 1 & 4.17 & 1 & 12.5 & \\
\hline TRAM & 53 & 59.55 & 33 & 57.89 & 16 & 66.67 & 4 & 50 & \\
\hline \multicolumn{10}{|l|}{ Complication rates } \\
\hline Hematoma & 9 & 10.23 & 3 & 5.26 & 5 & 20.83 & 1 & 14.29 & 0.1005 \\
\hline Seroma & 2 & 2.35 & 2 & 3.77 & 0 & 0 & 0 & 0 & 0.5388 \\
\hline Capsular Contracture & 2 & 2.25 & 2 & 3.51 & 0 & 0 & 0 & 0 & 0.5631 \\
\hline Total flap loss & 1 & 1.12 & 0 & 0 & 1 & 4.17 & 0 & 0 & 0.2542 \\
\hline Infection & 7 & 7.95 & 5 & 8.77 & 2 & 8.7 & 0 & 0 & 0.6836 \\
\hline Fat necrosis & 10 & 11.24 & 8 & 14.04 & 2 & 8.33 & 0 & 0 & 0.4354 \\
\hline
\end{tabular}

Abbreviations: DIEP, deep inferior epigastric artery perforator; SIEA, superficial inferior epigastric artery; TUG, transverse upper gracilis; TRAM, transverse rectus abdominus myocutaneous.

${ }^{a}$ Statistical significance.

batients with prior history of smoking. No patients in this study endorsed active smoking at time of surgery.

for the no skin paddle group compared with the large elliptical skin paddle group. MDs and NMDs reported that the areola skin paddle and no skin paddle groups "significantly decrease the prominence of visible scars" compared with the large and small elliptical paddles. Both MDs and NMDs rated overall scar appearance for all groups as "good-excellent" greater than $30 \%$ of the time and "fair-good" greater than $55 \%$ of the time for the elliptical paddle groups. While "poor" scar ratings were overall low for all groups, NMDs rated the areola paddle and the no skin paddle appearances as "poor" twice as often as MDs. In addition, NMDs rated smaller skin paddles (areola and no skin paddle) as having "poor" scar appearances twice as often as larger skin paddles (large and small elliptical paddles).

\section{Discussion}

Advances in autogenous breast reconstruction techniques have improved their functional outcomes yet aesthetic outcomes remain largely dependent on the mastectomy and the quantity/quality of preserved breast soft tissues. Although implicit in the process of breast reconstruction, factors that influence the mastectomy type and their effect on subsequent autologous skin paddle design remain largely undescribed.

Currently, there exists no standardized classification system or nomenclature for mastectomy type and their resultant skin paddle orientation. Several authors have discussed the importance of the mastectomy scar pattern on final breast outcome in both autologous and implant based breast reconstruction..$^{9}$ Tan et al described a classification system for different mastectomy incisions and skin paddle designs specific to the Asian patient population. ${ }^{10}$ This classification consists of a "racquet handle" incision and a periareolar incision alone or with adjacent skin excision or distant skin paddle. While useful, this classification is not applicable to the general population. Bourne et al describes three incision patterns used in autologous breast reconstruction following skin-sparing mastectomies: racquet, wise, and vertical incisions. However, this does not address patients with more extensive mastectomies and larger skin deficits. ${ }^{11}$ 
In performing autogenous breast reconstruction, the decision regarding native skin replacement and/or addition of new skin to the mastectomy defect is dependent on multiple factors. The general indications are for restorative and/or for monitoring purposes. For example, in nipple-sparing mastectomy, all native skin is preserved yet a small portion of transferred skin is maintained for perfusion monitoring in the immediate postoperative period and is typically excised in a subsequent procedure. In modified radical mastectomy, the nipple areolar complex and a portion of native breast skin is excised and a larger skin paddle is required. The need for a large restorative paddle was indeed identified in our study as all patients who underwent modified radical mastectomy received large or small elliptical skin paddles.

Presence of radiation injury or need for future radiotherapy is a critical consideration in autologous reconstruction. Radiation to the native breast skin results in microvascular circulatory changes, replacement of soft tissue with dense fibrotic tissue, and may lead to changes in skin quality and reconstruction success. ${ }^{12-14}$ Due to these changes, use of autologous reconstruction to replace radiation damaged soft tissues and performance of delayed breast reconstruction after completion of radiotherapy is becoming a more common practice. ${ }^{15,16}$ Despite the above, there has been controversy in the literature concerning the timing of breast reconstruction in patients receiving post mastectomy radiotherapy (PMRT). Several authors report that immediate breast reconstruction with subsequent radiotherapy has similar complication rates for reconstruction delayed until after radiotherapy is completed. However, current evidence suggests an aesthetic benefit to delayed breast reconstruction in the face of PMRT. ${ }^{17}$ For this reason, it is the practice of the senior author to delay autologous breast reconstruction until after PMRT has been completed.

In our study, patients with circumstances leading to increased scar tissue (radiation skin injury and delayed breast reconstruction) were more likely to require larger skin paddles. Delayed breast reconstruction was employed in $80.9 \%$ ( $n=72$ ) of the breasts in our study. Of these, $45.8 \%$ $(n=33)$ received prereconstruction radiation and $97.2 \%$ $(n=70)$ received large elliptical $(n=55)$ and small elliptical $(n=15)$ skin paddles. Approximately $20 \%$ of our patients underwent immediate breast reconstruction with only $64.7 \%$ $(n=11)$ receiving elliptical skin paddles and only $5.9 \%$ $(n=1)$ receiving prereconstruction radiation.

Patients with high BMI were also found to require larger skin paddles. These patients have larger breast volumes and, therefore, larger skin requirements. High BMI patients without radiation induced skin changes that receive immediate reconstruction often have macromastia and redundant skin, and it is counterintuitive that they would require a large skin paddle. However, this excess skin is often of poor quality and/ or vascularity, and the surgeon has the decision to either apply reduction pattern excision techniques versus replacement of this skin with free tissue transfer. In the latter, there is a larger relative skin deficiency compared with those patients with lower BMI (and likely smaller breasts), thus requiring larger skin paddles. Our results support this as patients who received large and small elliptical paddles had a mean BMI over 30 versus a mean BMI of 26 in those who received an areolar paddle or no paddle at all.

Our study is not without limitations. First, all but one of our patients received a variation of abdominal free tissue transfer. However, we do not predict that tissue transfers from other areas would change this classification system as the resulting skin paddle and scar pattern are primarily based on the health of the mastectomy flaps and various patient factors rather than the donor site. In addition, one physician at our institution performed all operations. Therefore, we cannot account for differences in technique that occur from surgeon to surgeon which may affect skin paddle size and design. Finally, specific limitations exist for the proposed SS3 group. There are several mastectomy flap incision designs possible in low BMI patients with large surface areas of healthy native breast skin following a mastectomy. This includes different orientations of the scars adjacent to the skin paddle. We have presented only transverse scars adjacent to the skin paddle in our SS3 group, as this is a common practice at our institution. In patients with excess skin, skin resection can be employed with resultant scar patterns similar to those in "wise" and vertical breast reductions. In addition, we did not include immediate oncoplastic reduction techniques, as these are already widely described in the literature and have standardized nomenclature. With regard to these scar patterns, Bourne et al showed no significant difference in shape or overall postoperative appearance in vertical skin resection pattern versus a transverse scar adjacent to the skin paddle. ${ }^{11}$

Several subsets of patients were excluded from this classification system. Patients who received regional autologous flaps, such as the pedicled latissimus dorsi myocutaneous flap (LDMC) were not included in this study, as this flap does not frequently have a sufficient skin component to allow near total breast skin replacement. Despite this, in lower BMI patients with smaller breast size, the skin paddle of the LDMC flap may assume a configuration similar to that described in our classification system (-Fig. 3). Autologous flaps following lumpectomies were not included since scar patterns following these procedures can vary widely based on tumor location or radiation injury. In addition, there are several incisions employed in nipple-sparing mastectomy (inframammary, vertical, and periareolar incisions) that were not used in this classification system. This is accounted for by the fact that the inferior periareolar scar with lateral extension is the preferred approach of our surgical oncologists due to improved visualization during the mastectomy. ${ }^{18}$ From the reconstructive standpoint, this incision allows for easy access to the internal mammary vessels while maintaining a satisfactory aesthetic outcome. Variations of the areola skin paddle (including circumareolar mastectomy incisions) and nipple-sparing mastectomy incisions not utilized in our classification system may be easily added to the classification system based on local practice preferences.

The survey validation of the classification system demonstrated both MDs and non-MDs could correctly categorize randomized patient pictures into their correct group over $75 \%$ 


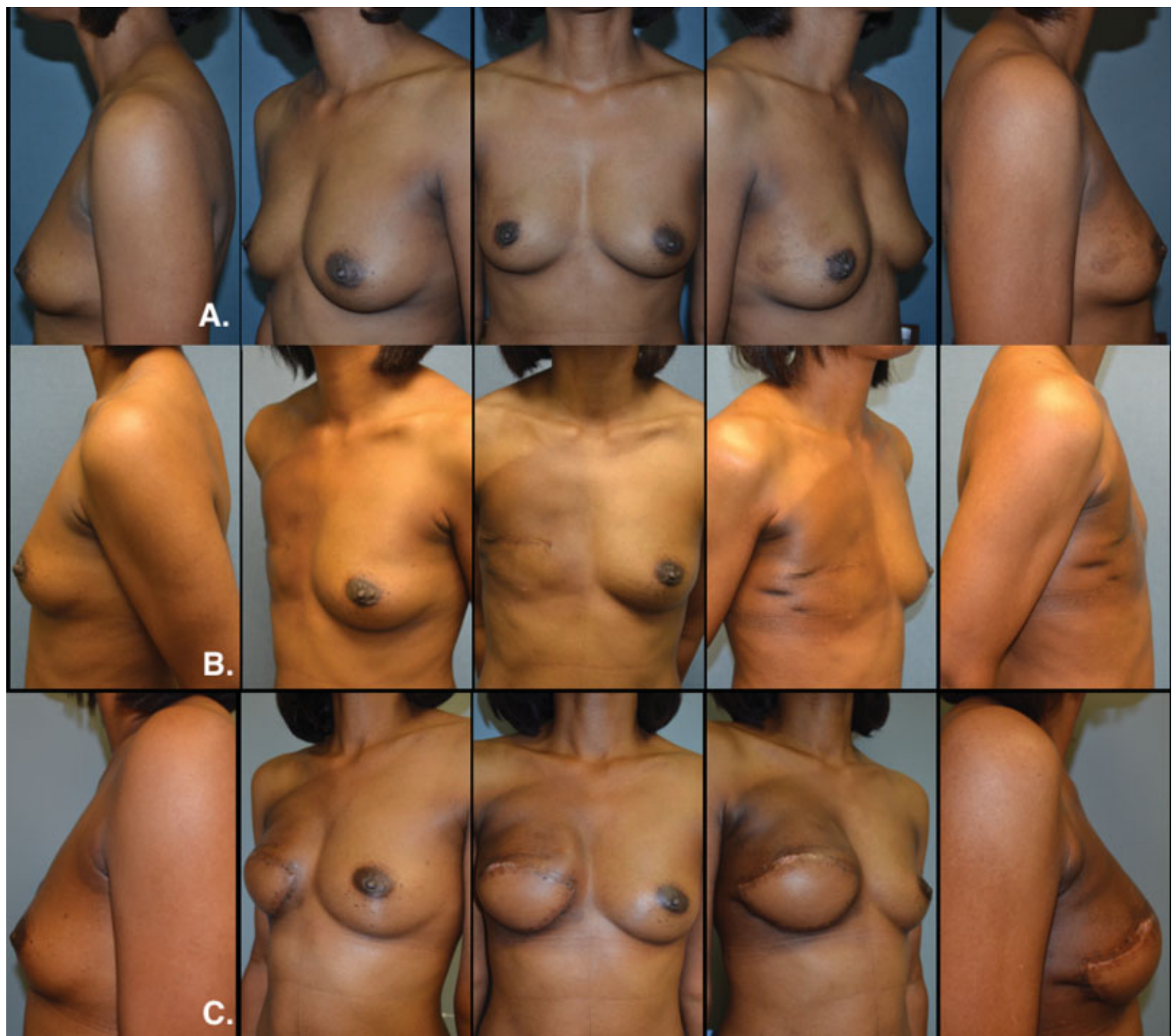

Fig. 3 Forty-year-old female patient who underwent right skin-sparing mastectomy and radiotherapy followed by delayed right breast reconstruction with latissimus dorsi myocutaneous (LDMC) flap. (A) Preoperative images following initial biopsy. (B) Postoperative images following right mastectomy/radiation. Note hyperpigmentation and soft tissue contraction of the right chest soft tissues. (C) Six-month postoperative images following LDMC and implant reconstruction. Note the skin paddle of the flap may assume an elliptical configuration similar to that described in Mastectomy type/skin paddle design scheme.

of the time. This suggests that the classification system is understood by and applicable to those with and without medical degrees. Physicians had an exceptionally high success rate in recognizing these common patterns, with $85.8 \%$ correct answers. This further suggests that this would be a particularly useful tool for communication between practitioners involved in treating breast disease that may require reconstruction (i.e., family practice, internal medicine, obstetrics and gynecology, general surgery, surgical oncology, etc.).

In our aesthetic survey, elliptical skin paddles were found to be too large three to five times more often than the areola paddle or no skin paddle groups, and most MDs found the areola and no skin paddle groups to be perfectly sized. This is no surprise as the areola skin paddle is positioned in the future location of nipple areolar complex reconstruction and the skin paddle maintained for monitoring in nipple-sparing mastectomy is usually small and removed in subsequent revision procedures.

There was discordance between MDs and NMDs on several important aesthetic components between groups. In addition to rating the elliptical skin paddles as too large, MDs also found them distracting to the NAC, while NMDs found that the elliptical skin paddles lead to a very natural NAC appearance. Overall, the no skin paddle group was rated the most natural around the NAC, which one would expect given these patients have preservation of their natural NAC. In contrast, NMDs rated results from the areola paddle and no skin paddle groups as poor more often than MDs. While the areola paddle and no skin paddle groups have less scar burden, it is not surprising that NMDs are less tolerant to scars adjacent to the NAC than 
those with more experience with common breast surgery scars patterns. Overall, all mastectomy patterns had high aesthetic ratings despite medical background of the observer. This is encouraging, and in instances where a patient is a good candidate for all options, physicians can be confident that no matter the decision, they have the opportunity to provide an acceptable, aesthetically pleasing result to their patient.

Standardized nomenclature for common results of autologous breast reconstruction will allow better communication amongst surgeons and will assist in counseling prospective reconstruction patients. Moreover, understanding patient factors that lead to certain scar/skin paddle patterns may allow for results that are more predictable for surgeons who perform autologous breast reconstruction. Patients may be better educated regarding their anticipated reconstruction result and potentially aid in the planning of their reconstruction based on their preferred scar pattern, if operative conditions allow. We predict this will improve overall patient outcomes and satisfaction with their reconstructed breast.

\section{Conclusion}

Patients with elevated BMI, delayed breast reconstruction or radiotherapy more commonly require larger skin paddles when autologous breast reconstruction is employed. This likely is due to the need for creation of larger breasts in the higher BMI patient population, as well as the need for more skin replacement, in the irradiated and/or scarred operative field. The mastectomy type/skin paddle patterns presented can be correctly utilized by both medical doctors and those without a medical degree and can provide a simple scheme to allow for improved patient education and communication among practitioners. All mastectomy pattern/skin paddle designs received high overall aesthetic ratings by both medical and nonmedical personnel.

\section{Conflict of Interest}

The authors have no conflicts of interest or source of funding to declare.

\section{References}

1 Eltahir Y, Werners LL, Dreise MM, et al. Quality-of-life outcomes between mastectomy alone and breast reconstruction: comparison of patient-reported BREAST-Q and other health-related quality-oflife measures. Plast Reconstr Surg 2013;132(02):201e-209e
2 Sisco M, Johnson DB, Wang C, Rasinski K, Rundell VL, Yao KA. The quality-of-life benefits of breast reconstruction do not diminish with age. J Surg Oncol 2015;111(06):663-668

3 Tsoi B, Ziolkowski NI, Thoma A, Campbell K, O'Reilly D, Goeree R. Safety of tissue expander/implant versus autologous abdominal tissue breast reconstruction in postmastectomy breast cancer patients: a systematic review and meta-analysis. Plast Reconstr Surg 2014;133(02):234-249

4 Schmauss D, Machens HG, Harder Y. Breast reconstruction after mastectomy. Front Surg 2016;2:71

5 Ramos-Gallardo G, Cuenca-Pardo J, Rodríguez-Olivares E, et al. Breast implant and anaplastic large cell lymphoma meta-analysis. J Invest Surg 2017;30(01):56-65

6 Brody GS, Deapen D, Taylor CR, et al. Anaplastic large cell lymphoma occurring in women with breast implants: analysis of 173 cases. Plast Reconstr Surg 2015;135(03):695-705

7 Nahabedian MY. Achieving ideal breast aesthetics with autologous reconstruction. . Gland Surg 2015;4(02):134-144

8 Oni G, Saint-Cyr M, Maia M, Colohan S, Rohrich RJ. Secondary techniques in breast reconstruction refinement: the periareolar advancement flap. Plast Reconstr Surg 2011;128(05):1015-1024

9 Dayicioglu D, Tugertimur B, Zemina K, et al. Vertical mastectomy incision in implant breast reconstruction after skin sparing mastectomy: advantages and outcomes. Ann Plast Surg 2016; 76(Suppl. (Suppl 4):S290-S294

10 Tan BK, Chim H, Ng ZY, Ong KW. Aesthetic design of skin-sparing mastectomy incisions for immediate autologous tissue breast reconstruction in asian women. Arch Plast Surg 2014;41(04):366-373

11 Bourne DA, Ahuja N, Gimbel ML. Analysis of the vertical mammaplasty design in skin-sparing mastectomy and immediate autologous reconstruction. J Plast Reconstr Aesthet Surg 2016;69(01):23-29

12 Tesselaar E, Flejmer AM, Farnebo S, Dasu A. Changes in skin microcirculation during radiation therapy for breast cancer. Acta Oncol 2017;56(08):1072-1080

13 Stone HB, Coleman CN, Anscher MS, McBride WH. Effects of radiation on normal tissue: consequences and mechanisms. Lancet Oncol 2003;4(09):529-536

14 Pestana IA, Campbell DC, Bharti G, Thompson JT. Factors affecting complications in radiated breast reconstruction. Ann Plast Surg 2013;70(05):542-545

15 Kronowitz SJ. Current status of autologous tissue-based breast reconstruction in patients receiving postmastectomy radiation therapy. Plast Reconstr Surg 2012;130(02):282-292

16 Javaid M, Song F, Leinster S, Dickson MG, James NK. Radiation effects on the cosmetic outcomes of immediate and delayed autologous breast reconstruction: an argument about timing. J Plast Reconstr Aesthet Surg 2006;59(01):16-26

17 D'Souza N, Darmanin G, Fedorowicz Z. Immediate versus delayed reconstruction following surgery for breast cancer. Cochrane Database Syst Rev 2011;((07):CD008674

18 Frey JD, Choi M, Salibian AA, Karp NS. Comparison of outcomes with tissue expander, immediate implant, and autologous breast reconstruction in greater than 1000 nipple-sparing mastectomies. Plast Reconstr Surg 2017;139(06):1300-1310 